\title{
Knockdown of miR-25 increases the sensitivity of liver cancer stem cells to TRAIL-induced apoptosis via PTEN/PI3K/Akt/Bad signaling pathway
}

\author{
XIAONING FENG ${ }^{1-3^{*}}$, JINGJIN JIANG $^{4 *}$, SHAOHUA SHI ${ }^{1-3}$, HAIYANG XIE ${ }^{1-3}$, \\ LIN ZHOU $^{1-3}$ and SHUSEN ZHENG ${ }^{1-3}$
}

${ }^{1}$ Key Laboratory of Combined Multi-Organ Transplantation, Ministry of Public Health and Key Laboratory of Organ Transplantation of Zhejiang Province; ${ }^{2}$ Collaborative Innovation Center for Diagnosis and Treatment of Infectious Diseases; ${ }^{3}$ Division of Hepatobiliary and Pancreatic Surgery, Department of Surgery,

The First Affiliated Hospital, School of Medicine, Zhejiang University; ${ }^{4}$ Department of VIP, The First Affiliated Hospital, College of Medicine, Zhejiang University, Hangzhou, Zhejiang 310003, P.R. China

Received August 18, 2016; Accepted October 19, 2016

DOI: $10.3892 /$ ijo.2016.3751

\begin{abstract}
Tumor necrosis factor (TNF)-related apoptosisinducing ligand (TRAIL) is considered as a promising anticancer agent, it induced apoptosis in cancer cells selectively without damaging the normal tissue cells. However, the resistance of cancer cells to TRAIL limits its application. Since the cancer stem cells (CSCs) are believed to be responsible for the treatment failure in multiple cancers including hepatocellular carcinoma (HCC), the aim of this study was to investigate the strategies to increase the sensitivity of liver cancer stem cells (LCSCs) to TRAIL. In the present study, we observed significant upregulation of miR-25 in LCSCs compared with the non-CSCs. Furthermore, we found that knockdown of miR-25 by its antisense oligonucleotide (anti-miR-25) significantly increased the sensitivity of LCSCs to TRAIL-induced apoptosis. The gene of phosphatase and tensin homologue (PTEN), which is a natural inhibitor of $\mathrm{PI} 3 \mathrm{~K}$, was found to be directly regulated by miR-25 in HepG2-CSCs. We demonstrated that knockdown of miR-25 increased the expression of PTEN. Mechanistically, inhibition of Bad phosphorylation, which is regulated by the PTEN/PI3K/AKT pathway, is essential for the functional roles of anti-miR-25 in HepG2-CSCs. In conclusion, our findings indicate that overexpression of miR-25 is associated with the low-sensitivity to TRAIL in LCSCs. Knockdown
\end{abstract}

Correspondence to: Dr Shusen Zheng, Division of Hepatobiliary and Pancreatic Surgery, Department of Surgery, The First Affiliated Hospital, School of Medicine, Zhejiang University, 79 Qingchun Road, Hangzhou, Zhejiang 310003, P.R. China

E-mail: hangzhoussz@163.com

${ }^{*}$ Contributed equally

Key words: liver cancer stem cells, TNF-related apoptosis-inducing ligand, miR-25, resistance, phosphatase and tensin homologue, PI3K/AKT of miR-25 may represent a potential strategy for increasing the efficacy of TRAIL by targeting the PTEN/PI3K/Akt/Bad signaling pathway.

\section{Introduction}

Hepatocellular carcinoma (HCC) represents the fifth most commonly diagnosed malignancy and the third leading cause of cancer-related death worldwide (1). Due to the extensive research efforts, understanding of the molecular mechanisms involved in the tumorigenic transformation of $\mathrm{HCC}$ has been clarified gradually. However, the molecular targets for HCC therapy remain insufficient (2). Recent studies suggest that human cancer can be considered as a stem cell disorder. According to these studies, tumors may be viewed as abnormal growth which is driven by a small population of cancer cells called cancer stem cells (CSCs) that are endowed with the ability of self-renewal $(3,4)$.

Previous studies have identified the $\mathrm{CD}_{133^{+}}$phenotype as the molecular marker of liver cancer stem cells (LCSCs) (5). The LCSCs were found to show a greater colony-forming efficiency and greater ability to form a tumor in vivo. In addition, the LCSCs are believed to be responsible for the high resistance to chemotherapeutic drugs (6). Therefore, to treat the HCC more effectively, it is imperative that LCSCs are specifically targeted.

Tumor necrosis factor (TNF)-related apoptosis inducing ligand (TRAIL) is a cytokine, which belongs to the TNF superfamily (7). As the TRAIL triggers apoptosis selectively in tumor cells without damaging the normal tissue cells, it has been tested in clinical trials for cancer therapy (8). However, unfortunately, some cancer cells (especially CSCs) are resistant to TRAIL and the mechanism of this resistance is not fully understood (9). Therefore, it is urgent to identify the mechanisms and take strategies to decrease the resistance of CSCs to TRAIL.

MicroRNAs (miRNAs), small functional RNAs of 19-25 nt, are a class of short non-coding RNA molecules and play 
important regulatory roles by binding to the target mRNAs at the 3'-untranslated region (3'-UTR) $(10,11)$. Increasing evidence shows that miRNAs have significant roles in a number of biological processes such as proliferation, differentiation, metabolism and apoptosis. Aberrant expression of miRNAs is associated with multiple human diseases including cancer $(12,13)$. In the present study, we found the miR-25 was dysregulated in LCSCs. We therefore investigated the relationship between the miR-25 and the treatment of TRAIL in LCSCs, which may serve as a potential target for HCC therapy.

\section{Materials and methods}

Animals. Nude mice (BALB/c, nu/nu; 4-5 week old; 18-23 g in weight) were obtained from the Shanghai Laboratory Animal Center (Shanghai, China). The animals were kept in ventilated, pathogen-free racks under a $12 \mathrm{~h}$ light-dark photoperiodicity with controlled humidity (50-60\%) and temperature $\left(22-24^{\circ} \mathrm{C}\right)$. The animals were kept with free access to food and water. Animal care and the experimental procedures in this study were approved by the Animal Care Committee of the First Affiliated Hospital, School of Medicine, Zhejiang University and complied with the recommendations of the Chinese guidelines for the care and use of laboratory animals.

Cell lines. The HCC cells lines of HepG2, Huh7, PLC and the normal liver cell line of L-O2 were obtained from the Institute of Biochemistry and Cell Biology, Chinese Academy of Sciences (Shanghai, China). Cells were cultured in DMEM basic medium containing 10\% fetal bovine serum (FBS) (both from Gibco, Grand Island, NY, USA) at $37^{\circ} \mathrm{C}$ in a humidified $5 \% \mathrm{CO}_{2}$ incubator. To evaluate the role of anti-miR-25 in HCC in vivo, the stable HepG2 cell line with miR-25 knockdown was generated. Briefly, we purchased the recombinant lentivirus contained miR-25 antisense nucleotide sequence from the Shanghai Genechem Co., Ltd. (Shanghai, China). The routine HepG2 cells were then transfected with $5 \times 10^{5}$ transducing units of lentivirus, and the cells were selected with $1 \mu \mathrm{g} / \mathrm{ml}$ puromycin for 2 weeks.

Measurement of miR-25 expression level. The expression level of miR-25 was measured by reverse transcription and quantitative real-time polymerase chain reaction (qRTPCR). Briefly, total RNA was extracted from the cells using TRIzol reagent (Invitrogen, Carlsbad, CA, USA) according to the manufacturer's instructions. The reverse transcription reaction for miR-25 was performed by using the One Step PrimeScript miRNA cDNA synthesis kit (Takara, Dalian, China). Subsequently, the qRT-PCR reaction was performed by SYBR ${ }^{\circledR}$ Premix Ex Taq ${ }^{\mathrm{TM}}$ II (Takara). The expression of miR-25 was determined using the $2^{-\Delta \Delta \mathrm{CT}}$ analysis method (14) taking the U6 snRNA as the internal control.

Identification and sorting of LCSCs and non-CSCs. HCC cells were collected and washed twice. Cell samples were then stained with anti-CD133-FITC (Miltenyi Biotec, Gladbach, Germany) for $20 \mathrm{~min}$ at room temperature. The percentages of $\mathrm{CD}_{133^{+}}$cells were analyzed and sorted as the LCSCs using the fluorescent-activated cell sorting equipment (Beckman Coulter, Brea, CA, USA).
Transfection. Cell-based experiments were carried out by transfection of $50 \mathrm{pmol} / \mathrm{ml}$ RNA oligonucleotides including miR-25 mimics, miR-25 antisense-oligonucleotides (anti-miR-25), control RNA oligonucleotides (miR-NC) and small interfering RNA of phosphatase and tensin homologue (PTEN) (PTEN siRNA). All of these RNAs were purchased from RiboBio Co., Ltd. (Guangzhou, China) and transfected into the cells using Lipofectamine $^{\mathrm{TM}} 2000$ reagent (Invitrogen) according to the manufacturer's instructions.

Cell viability assay. The sorted LCSCs and non-CSCs were seeded in 96-well plates at $5 \times 10^{3}$ cells/well and transfected with $50 \mathrm{pmol} / \mathrm{ml}$ RNA oligonucleotides. Twenty-four hours posttransfection, cells were incubated in different concentrations of TRAIL for $48 \mathrm{~h}$. Subsequently, $20 \mu \mathrm{l}$ 3-(4,5-dimethylthiazol-2-yl)-2,5-diphenyltetrazolium bromide (MTT) $(5 \mathrm{mg} / \mathrm{ml}$; Sigma-Aldrich, St. Louis, MO, USA) was added to the medium and incubated at $37^{\circ} \mathrm{C}$ for $4 \mathrm{~h}$ followed by lysing in dimethyl sulfoxide at room temperature for $30 \mathrm{~min}$. The absorbance in each well was measured at $570 \mathrm{~nm}$ using a microplate reader (Sunrise Microplate Reader; Tecan, Männedorf, Switzerland).

Luciferase reporter assay. Fragment of PTEN 3'-UTR containing the seed region of miR-25 was cloned into the pGL3 Luciferase Reporter Vectors (Promega, Madison, WI, USA) named pGL3-PTEN. The mutant PTEN reporter was created by mutating the seed regions of the miR-25 binding sites (GUGCAAU) by using the site-directed mutagenesis kit (Takara, Shiga, Japan) named pGL3-mutant PTEN. For luciferase reporter assay, LCSCs were seeded in 48-well plates followed by co-transfection with $50 \mathrm{pmol} / \mathrm{ml} \mathrm{miR}-25 \mathrm{mimics}$ or inhibitors together with $2 \mu \mathrm{g} / \mathrm{ml}$ Firefly luciferase reporters and $100 \mathrm{ng} / \mathrm{ml}$ Renilla luciferase pRL-TK vector (Promega). Luciferase activity was measured $48 \mathrm{~h}$ after transfection by using the Dual-Luciferase Reporter assay system (Promega) according to the manufacturer's instructions.

Mitochondria isolation. For detecting the release of cytochrome $c$, second mitochondria derived activator of caspase/directIAP binding protein with low $\mathrm{pI}$ (Smac/DIABLO) and apoptosis-inducing factor (AIF) in cytoplasm, mitochondria of LCSCs were isolated using the Mitochondria Isolation kit for Mammalian Cells (Thermo Fisher Scientific, Rockford, IL, USA) according to the manufacturer's guidance.

Immunoprecipitation. Cells were lysed in RIPA buffer and centrifuged at $12,000 \mathrm{x} \mathrm{g}$ for $10 \mathrm{~min}$. The resulting supernatants were collected and incubated with primary antibody of Bad (Santa Cruz Biotechnology, Inc., Santa Cruz, CA, USA) overnight at $4^{\circ} \mathrm{C}$. Subsequently, the protein A agarose beads were added and incubated for $2 \mathrm{~h}$. After washing the beads with cold RIPA buffer, the co-precipitated proteins were removed from the beads by boiling in sodium dodecyl sulfate (SDS) sample buffer.

Western blot analysis. Fifty micrograms of the extracted and the co-precipitated proteins were separated by $10 \%$ SDS-polyacrylamide gel electrophoresis (PAGE) and transferred to a PVDF membrane (Millipore, Billerica, MA, USA). The membrane was then blocked with $5 \%$ non-fat milk 

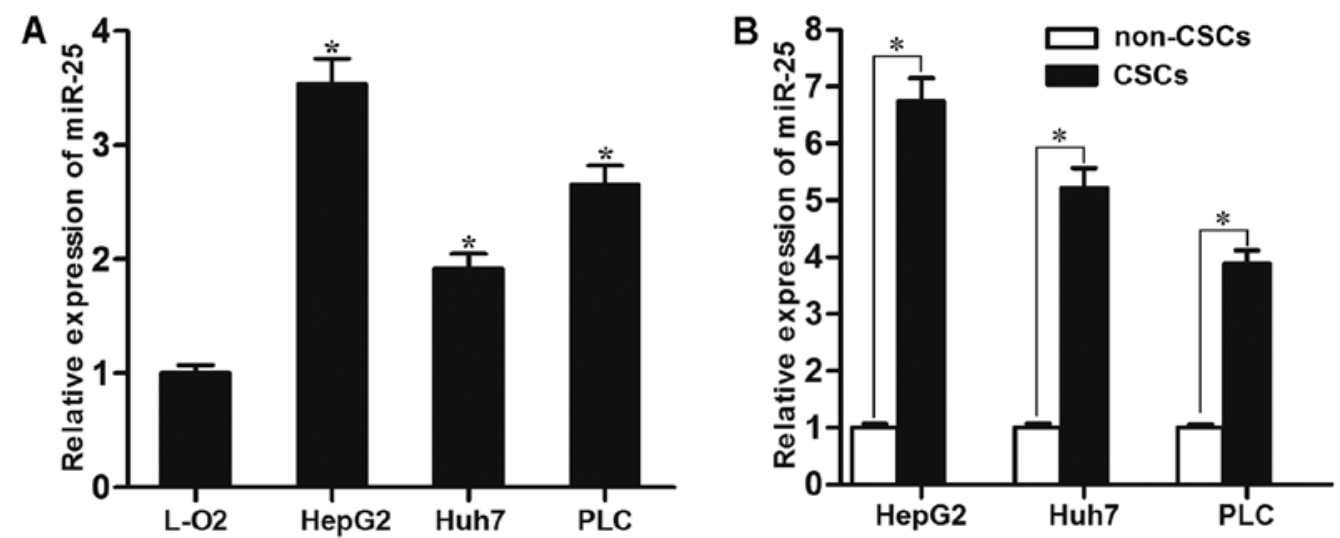

Figure 1. miR-25 is overexpressed in liver cancer stem cells (LCSCs). (A) miR-25 expression levels were examined by quantitative real-time polymerase chain reaction (qRT-PCR) in normal liver cells (L-O2, as control group) and three hepatocellular carcinoma (HCC) cell lines (HepG2, Huh7 and PLC). ${ }^{*} \mathrm{P}<0.05$ vs. control group. (B) The LCSC population was sorted as $\mathrm{CD} 133^{+}$cells, and the CD133-/low cells were sorted as the non-cancer stem cells (non-CSCs) in $\mathrm{HCC}$ cell lines. ${ }^{*} \mathrm{P}<0.05$.

and incubated with various antibodies followed by incubation of horseradish peroxidase-conjugated antibody (Santa Cruz Biotechnology, Inc.). Signals were detected using enhanced chemiluminescence (ECL) reagents (Thermo Fisher Scientific) according to the manufacturer's guidance.

Detection of apoptosis and reactive oxygen species (ROS). LCSCs were treated with RNAs and TRAIL. Then, the cells were stained with PI and Annexin V (Sigma-Aldrich) for apoptosis detection and dihydroethidium (DHE; Molecular Probes, Camarillo, CA, USA) for measurement of ROS. Both apoptosis and ROS were detected on the flow cytometry according to the manufacturer's guidance, respectively.

Tumor growth in nude mice. An equal number $\left(5 \times 10^{6}\right)$ of HepG2 cells transfected with lentivirus anti-miR-25 or lentivirus control were harvested and washed. The experimental animals were divided into four groups ( $8 \mathrm{mice} / \mathrm{group}$ ). For xenografts, two groups of mice were subcutaneously injected with lentivirus control HepG2 cells (for lenti control group and TRAIL group), and two groups of mice were subcutaneously injected with lentivirus anti-miR-25 HepG2 cells (for anti-miR-25 group and TRAIL plus anti-miR-25 group). The tumor volume $(\mathrm{V})$ was calculated based on the following equation: $1 / 2 \times$ length $\mathrm{x}$ width ${ }^{2}$. The mice in TRAIL group and TRAIL plus anti-miR-25 group received intraperitoneal (i.p.) injections of $40 \mu \mathrm{g} / \mathrm{kg}$ TRAIL per two days when the tumors reached a mean volume of $100 \mathrm{~mm}^{3}$ (15). The tumor formation was monitored every three days using calipers. Nude mice were euthanized at the experimental end-point (31 days postinjection).

To measure the population of CSCs in tumor tissues, we purified the cells from the tumor. Briefly, the tumor xenografts were cut and digested with $1 \mathrm{mg} / \mathrm{ml}$ of collagenase type III (5 ml/g tissue; Worthington Biochemical, Lakewood, NJ, USA) in $10 \%$ FBS containing DMEM medium at $37^{\circ} \mathrm{C}$ for $2 \mathrm{~h}$. The resulting cells were then stained with anti-CD133-FITC for 20 min before analyzing on the FACS vantage.

Statistical analysis. The data are presented as the mean \pm standard deviation and carried out by at least three independent experiments. Statistical analysis was performed by Student's t-test using SPSS 15.0 software. Values of $\mathrm{P}<0.05$ were considered statistically significant.

\section{Results}

miR-25 is overexpressed in LCSCs. To investigate the role of miR-25 in HCC, its expression levels between normal liver cell line and HCC cell lines were compared by qRT-PCR. The expression of miR-25 was significantly higher in HCC cell lines than that in the L-O2 cells (Fig. 1A). We further evaluated the expression of miR-25 in the CSCs of HepG2, Huh7 and PLC, as well as in the non-CSCs of these cell lines. As shown in Fig. 1B, miR-25 was upregulated overall in all tested HCC cell lines. Taken together, these results suggested that the upregulation of miR-25 may be involved in LCSCs.

miR-25 is associated with the sensitivity of LCSCS to TRAIL in vitro. In order to evaluate the sensitivity of LCSCs and non-CSCs to TRAIL, we sorted them in HepG2 cell line using the anti-CD133 antibody. Subsequently, the HepG2-CSCs and non-CSCs were treated with different concentrations of TRAIL. The sensitivity to TRAIL was significantly lower in HepG2-CSCs than that in the non-CSCs (Fig. 2A). The results suggest the high resistance of LCSCs to TRAIL. To evaluate the role of miR-25 in LCSCs, we transfected the HepG2-CSCs and non-CSCs with the miR-25 mimic or inhibitors. Transfection of miR-25 mimics significantly upregulated the expression level of miR-25 in both the HepG2-CSCs and non-CSCs, whereas the expression of miR-25 was significantly downregulated due to the transfection of anti-miR-25 (Fig. 2B). Interestingly, the flow cytometric analysis showed that the percentage of $\mathrm{CD} 133^{+}$ HepG2-CSCs population was increased after the single treatment of TRAIL. On the other hand, although changing the expression of miR-25 did not influence the percentage of HepG2-CSCs population significantly, the anti-miR-25 significantly inhibited the effect of TRAIL on enriching the HepG2-CSCs population, whereas the miR-25 mimics even increased the enrichment of HepG2-CSCs population induced by TRAIL (Fig. 2C and D). We consider that the treatment of TRAIL alone probably killed the TRAIL-sensitive non-CSCs, 

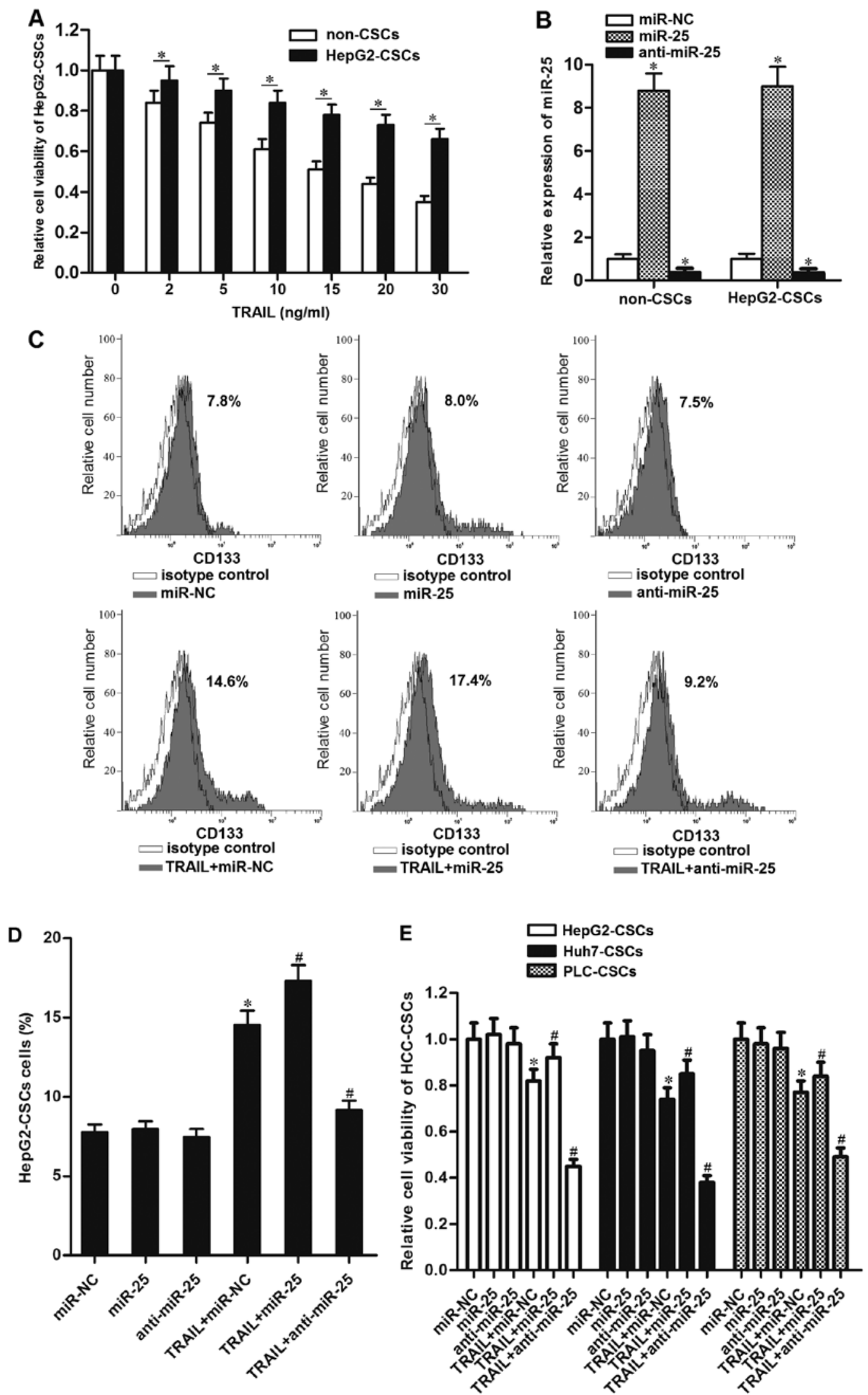

Figure 2. miR-25 is associated with the sensitivity of liver cancer stem cells (LCSCs) to tumor necrosis factor (TNF)-related apoptosis inducing ligand (TRAIL) in vitro. (A) HepG2-CSCs and non-CSCs were sorted and subsequently treated with different concentrations of TRAIL. The sensitivity of these cells was evaluated by MTT assay. "P<0.05. (B) After transfection with miR-NC, miR-25 and anti-miR-25, the expression of miR-25 in the HepG2-CSCs and non-CSCs were monitored using qRT-PCR. ${ }^{*} \mathrm{P}<0.05$ vs. miR-NC group. (C) The effect of miR-25, anti-miR-25 and TRAIL (10 ng/ml) on changing the percentage of the HepG2-CSCs population was evaluated by flow cytometry using CD133 antibody. (D) miR-25 inhibitors inhibited the effect of TRAIL on enriching the HepG2-CSCs population in HepG2 cell line. ${ }^{*} \mathrm{P}<0.05$ vs. miR-NC group; ${ }^{~} \mathrm{P}<0.05$ vs. TRAIL+miR-NC group. (E) After treatment with miR-25, anti-miR-25 and TRAIL ( $10 \mathrm{ng} / \mathrm{ml}$ ), the cell viability of HepG2-CSCs, Huh7-CSCs and PLC-CSCs were measured by MTT assay. "P<0.05 vs. miR-NC group; ${ }^{\text {"P }}<0.05$ vs. TRAIL+miR-NC group. 

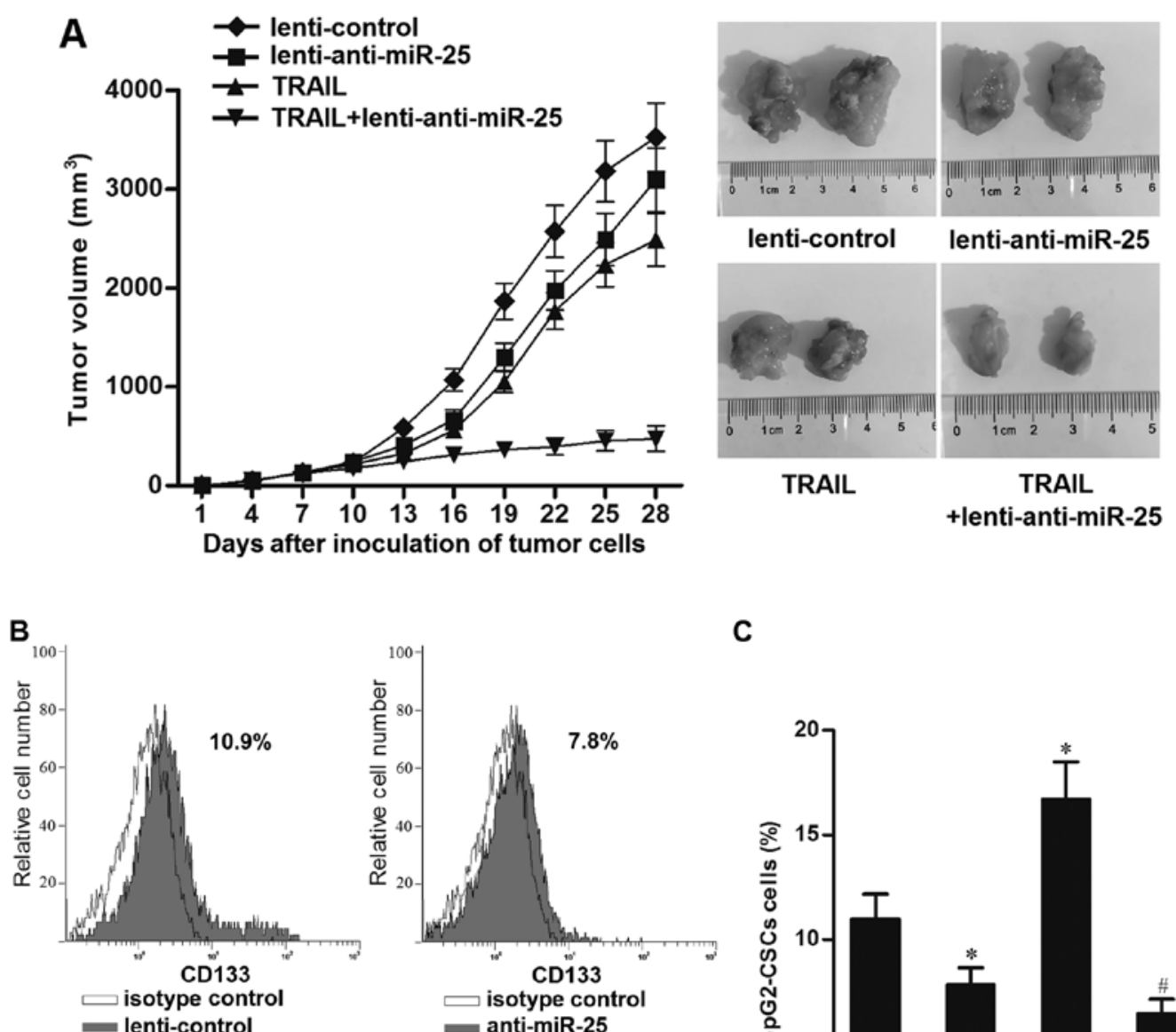

C
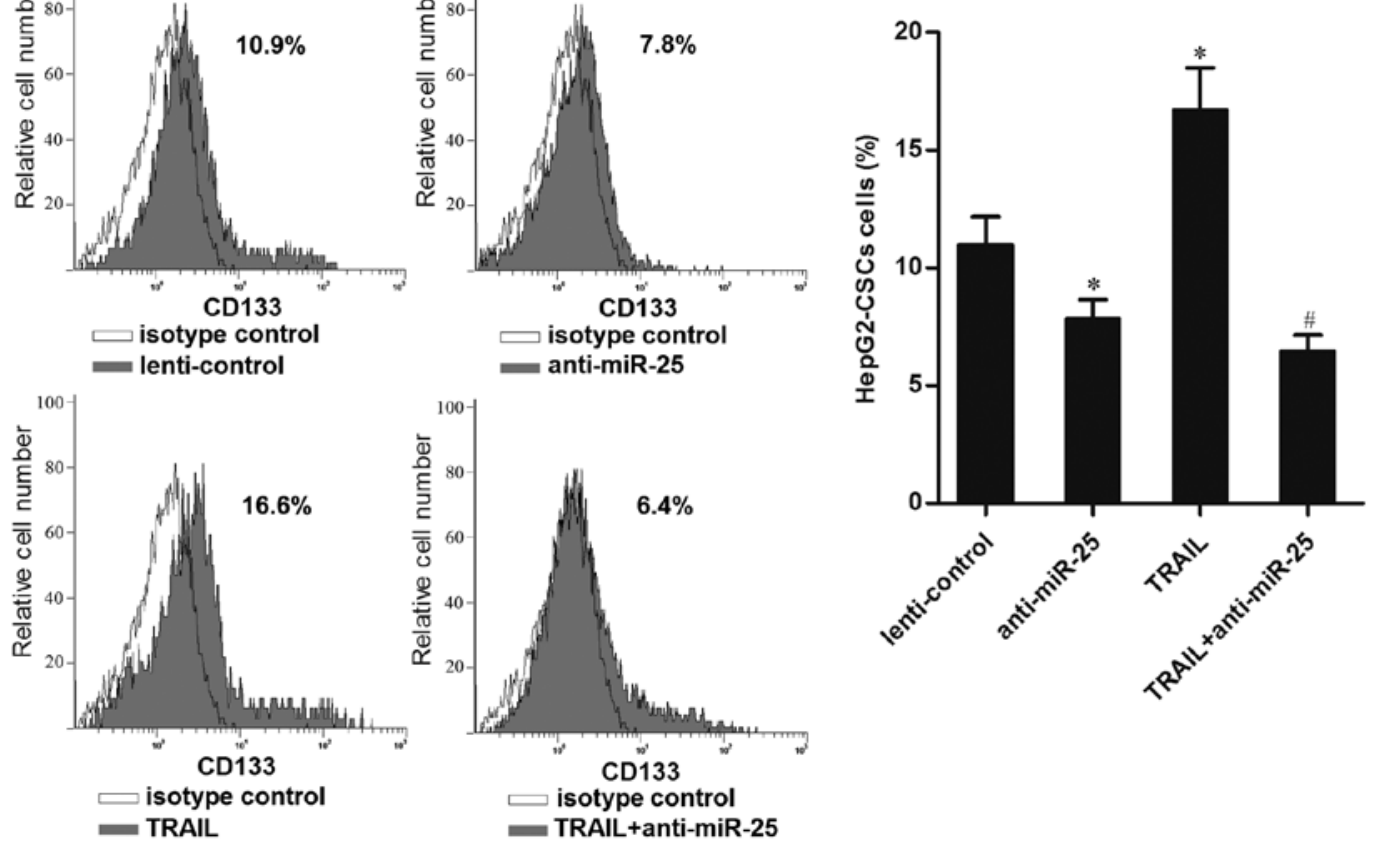

Figure 3. Knockdown of miR-25 increases the antitumor effect of tumor necrosis factor (TNF)-related apoptosis inducing ligand (TRAIL) on hepatocellular carcinoma (HCC) in vivo. (A) Volumes of HepG2 tumors in vivo were detected every three days with or without anti-miR-25 transfection and TRAIL treatment. (B) The population of CSCs in tumor cells in vivo was detected by flow cytometry. (C) Knockdown of miR-25 inhibited the effect of TRAIL on enriching the HepG2-CSCs population in vivo. ${ }^{*} \mathrm{P}<0.05$ vs. lenti-control group; ${ }^{\#} \mathrm{P}<0.05$ vs. TRAIL group.

whereas the TRAIL-resistant HepG2-CSCs survived under the TRAIL treatment. To demonstrate the effect of miR-25 on the sensitivity of LCSCs to TRAIL, we next performed a cell viability assay. The HCC-CSCs (HepG2-CSCs, Huh7CSCs and PLC-CSCs) transfected with anti-miR-25 showed significantly higher sensitivity to TRAIL than those in the miR-NC group (Fig. 2E). However, the cells transfected with miR-25 mimics showed the opposite effect on the sensitivity of TRAIL compared with those in the miR-NC groups. Taken together, these results indicated that knockdown of miR-25 could increase the sensitivity of LCSCs to TRAIL-induced cell death in vitro.

Knockdown of miR-25 increases the antitumor effect of TRAIL on HCC in vivo. To investigate the effect of miR-25 inhibitors on TRAIL treatment in vivo, a total of $5 \times 10^{6}$ of HepG2 cells transfected with lentivirus anti-miR-25 or lentivirus control were inoculated to the nude mice. Then, the tumor volumes were calculated every three days. The results showed that the growth of HepG2 cells transfected with lentivirus antimiR-25 was significantly slower than the cells transfected with lentivirus control when they were treated with equal dose of TRAIL in vivo (Fig. 3A). Furthermore, the results of flow cytometry indicated that the percentage of CSC population in lentivirus control tumor tissues was significantly higher than the lentivirus anti-miR-25 tumor tissues when the mice were treated with equal dose of TRAIL in vivo (Fig. 3B and C). Taken together, these results indicated that knockdown of miR-25 could enhance the curative effect of TRIAL and increase the sensitivity of LCSCs to TRAIL in vivo. 
A

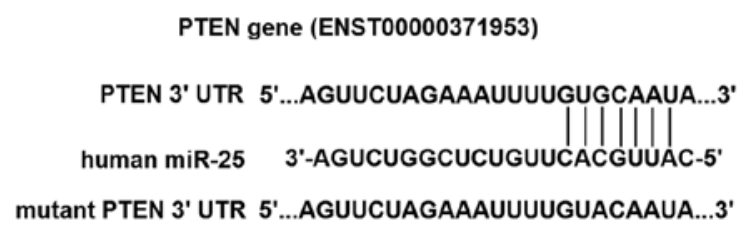

C

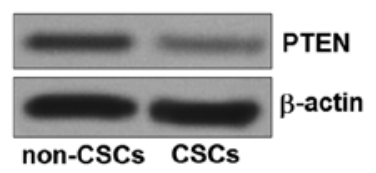

D

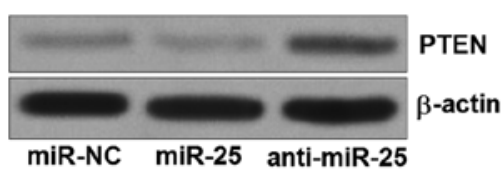

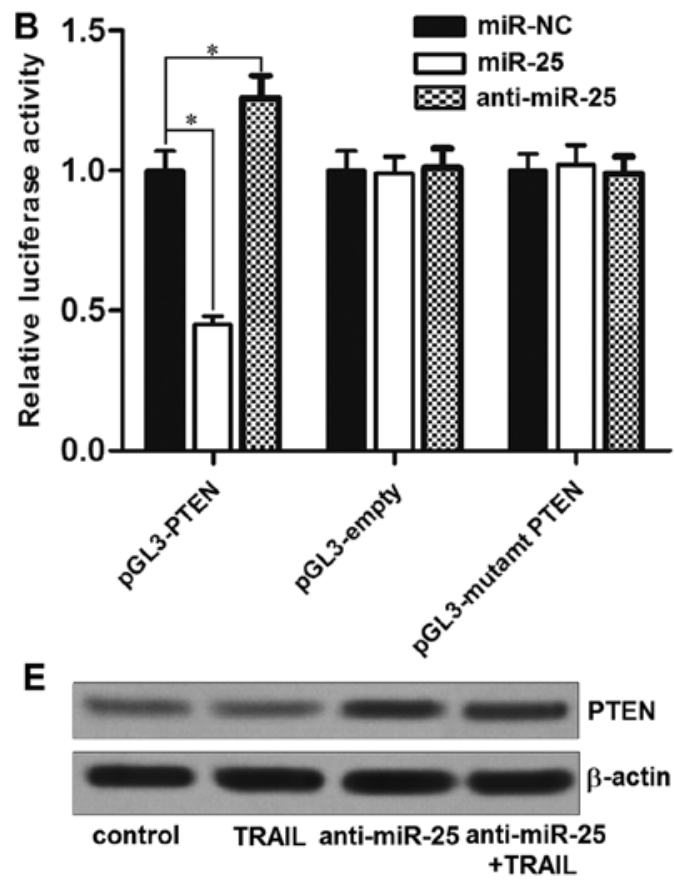

Figure 4. Phosphatase and tensin homologue (PTEN) is the target of miR-25 in HepG2-CSCs. (A) The putative miR-25 binding sites on 3'-untranslated region (3'-UTR) of PTEN mRNA was predicted by the TargetScan, miRanda and PicTar databases. (B) After transfection, the luciferase activity was detected using dual-luciferase reporter assay system according to the manufacturer's instruction. Each value is evaluated by the relative luciferase activity of Firefly to Renilla. ${ }^{*} \mathrm{P}<0.05$. (C) The expression of PTEN in HepG2-CSCs and non-CSCs was evaluated by western blot analysis. (D) The effect of miR-25 and anti-miR-25 on regulating the expression of PTEN in HepG2-CSCs. (E) Knockdown of miR-25 increased the expression of PTEN HepG2 cells in vivo.

PTEN is the target of miR-25 in HepG2-CSCs. To understand how miR-25 regulated the sensitivity of TRAIL to LCSCs, three different public databases (TargetScan, miRanda and PicTar) were used. All of these databases showed a prediction that a highly conserved sequence in the 3'-UTR of the PTEN mRNA was targeted by miR-25 (Fig. 4A). To investigate whether miR-25 directly targets the PTEN mRNA, we cloned the PTEN 3'-UTR sequence into the pGL3 luciferase reporter vectors. The results of dual luciferase reporter assays showed that co-transfection of miR-25 mimics and luciferase reporter with wild-type of PTEN 3'-UTR significantly decreased the luciferase activities, whereas the anti-miR-25 increased the luciferase activities. Moreover, the mutant or empty plasmids exhibited no response to miR-25 or anti-miR-25 (Fig. 4B). These results suggest that miR-25 directly regulates the PTEN in HepG2-CSCs. Consistent with this, we found the expression level of PTEN was significantly lower in HepG2-CSCs than that in the corresponding non-CSCs (Fig. 4C), because the expression of miR-25 is overexpressed in HepG2-CSCs. To confirm that miR-25 regulates the expression of PTEN, the PTEN protein was measured after the miR-25 or anti-miR-25 was transfected into HepG2-CSCs. Transfection of miR-25 decreased the expression of PTEN (Fig. 4D). Whereas, the transfection of anti-miR-25 increased the expression of PTEN. In addition, we detected the expression of PTEN in the lenticontrol tumor tissues (mice in control group and TRAIL group) and lenti anti-miR-25 tumor tissues (mice in anti-miR-25 group and anti-miR-25 plus TRAIL group) in vivo. We found that the protein levels of PTEN in miR-25 knockdown tumor cells were significantly increased compared with the control tumor cells (Fig. 4E). Taken together, we demonstrated that the PTEN gene is a functional target of miR-25 in HCC.
Knockdown of miR-25 increases the sensitivity of HepG2-CSCs to TRAIL through PTEN pathway. To investigate the role of PTEN in the anti-miR-25 promoted cell death in HepG2-CSCs, we knocked down the expression of PTEN by its specific siRNA, and the transfection efficiency of PTEN siRNA is shown in Fig. 5A. We observed that although the anti-miR-25 significantly enhanced the cytotoxicity of TRAIL to HepG2-CSCs, the promotion of anti-miR-25 was significantly inhibited when the PTEN siRNA was introduced (Fig. 5B). Furthermore, as the anti-miR-25 inhibited the enrichment of HepG2-CSC population induced by TRAIL, transfection of PTEN siRNA abolished the effect of anti-miR-25 in HepG2 cells (Fig. 5C and D). Taken together, these results indicate that the anti-miR-25 increases the sensitivity of HepG2-CSCs to TRAIL by upregulating the expression of PTEN.

Knockdown of miR-25 increases the sensitivity of HepG2CSCs to TRAIL through apoptosis pathway. We observed that although the anti-miR-25 did not induce apoptosis of HepG2CSCs directly, it significantly enhanced the effect of TRAIL on inducing the cell death through the apoptosis pathway. In addition, the transfection of PTEN siRNA rescued the HepG2CSCs from apoptosis induced by the combination with TRAIL and anti-miR-25 (Fig. 6A). Furthermore, we found knockdown of miR-25 promoted the activation of caspase- 9 and caspase- 3 induced by the TRAIL treatment in the HepG2-CSCs. Moreover, the transfection of PTEN siRNA inhibited the cleavage of these caspases induced by the combination with TRAIL and anti-miR-25 (Fig. 6B). These results indicate that knockdown of miR-25 in HepG2-CSCs enhanced the TRAILinduced apoptosis through the PTEN pathway. 
A

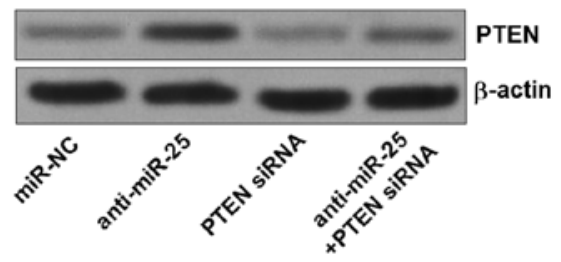

B gू

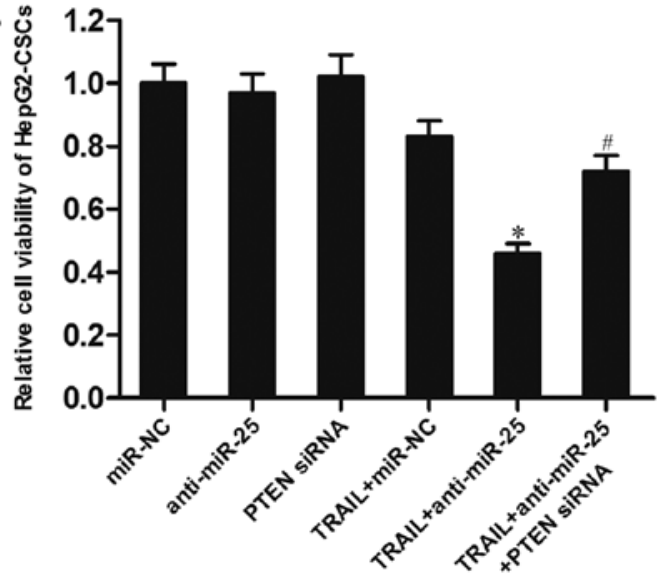

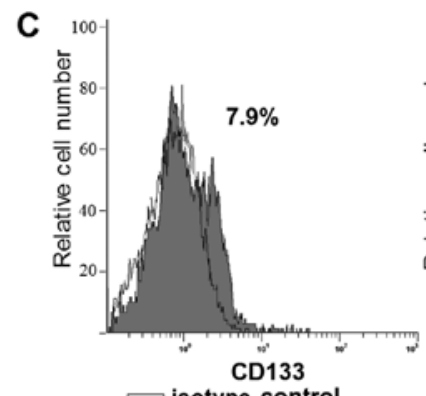

isotype contro miR-NC

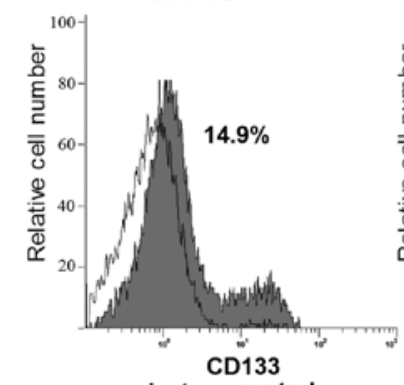

isotype contro

TRAIL+miR-NC

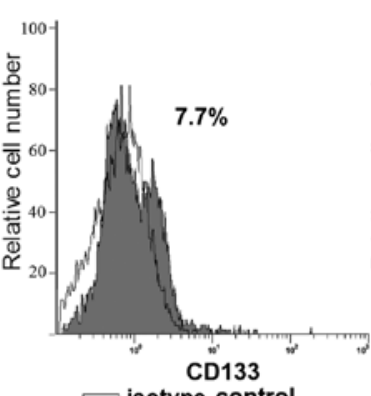

$\square$ isotype control anti-miR-25

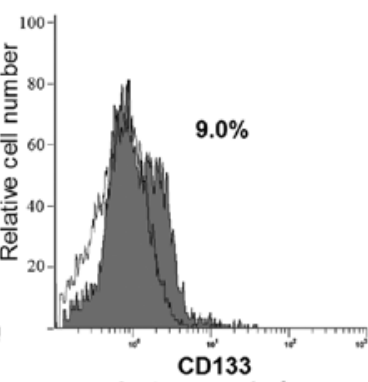

$\square$ isotype control

TRAIL+anti-miR-25

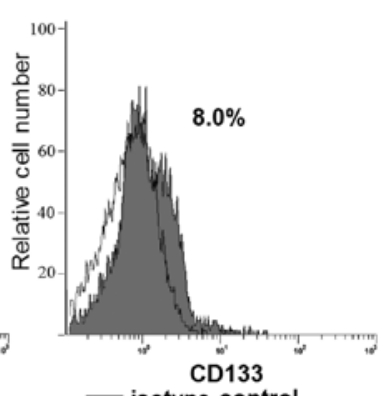

$\square$ isotype contro

PTEN sIRNA

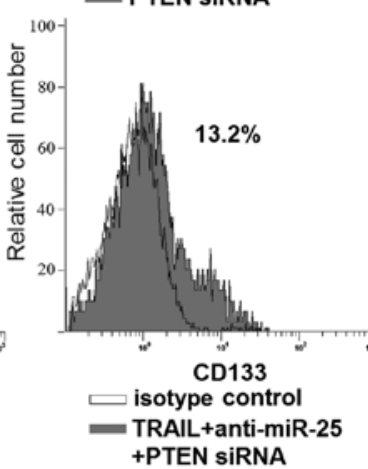

D

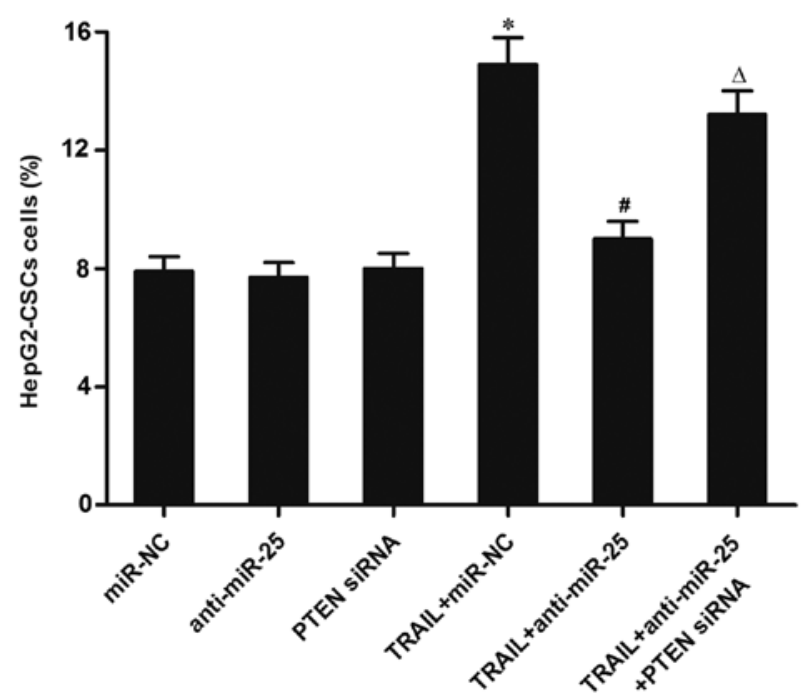

Figure 5. Phosphatase and tensin homologue (PTEN) pathway is essential to the promotion of anti-miR-25 on the tumor necrosis factor (TNF)-related apoptosis inducing ligand (TRAIL)-induced cell death in HepG2-CSCs. (A) The upregulation of PTEN induced by anti-miR-25 was inhibited by PTEN siRNA in HepG2-CSCs. (B) The promotion of anti-miR-25 on the TRAIL (10 ng/ml)-induced cell death was inhibited by the PTEN siRNA in HepG2-CSCs. ${ }^{*} \mathrm{P}<0.05$ vs. TRAIL+miR-NC group; ${ }^{\#} \mathrm{P}<0.05$ vs. TRAIL + anti-miR-25 group. (C) The effect of anti-miR-25, TRAIL and PTEN siRNA on changing the percentage of the HepG2-CSCs population was evaluated by flow cytometry using CD133 antibody. (D) The inhibition of anti-miR-25 on the TRAIL (10 ng/ml)-induced enrichment of HepG2-CSCs population was abolished by the PTEN siRNA in HepG2 cells. *P<0.05 vs. TRAIL + miR-NC group; ${ }^{\#} \mathrm{P}<0.05$ vs. TRAIL + anti-miR-25 group. 

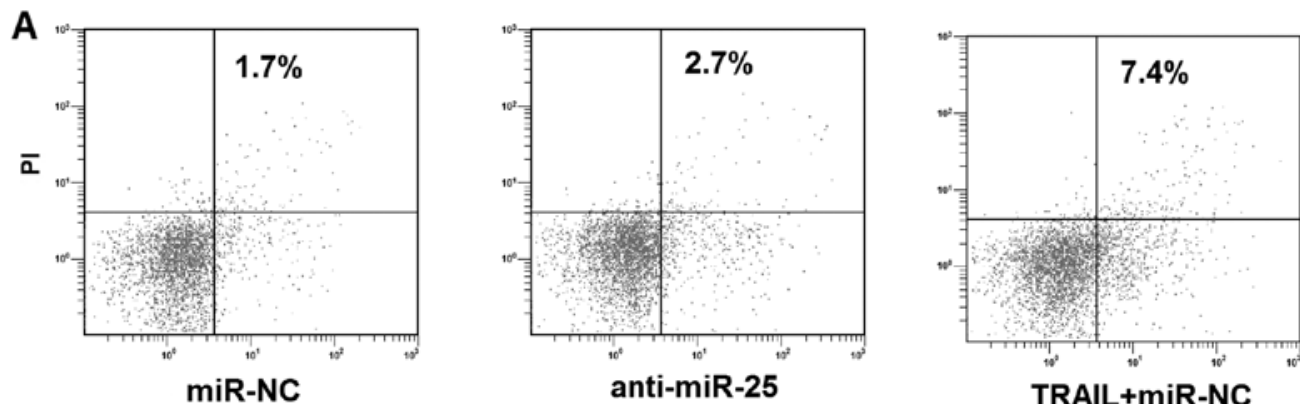

Annexin V
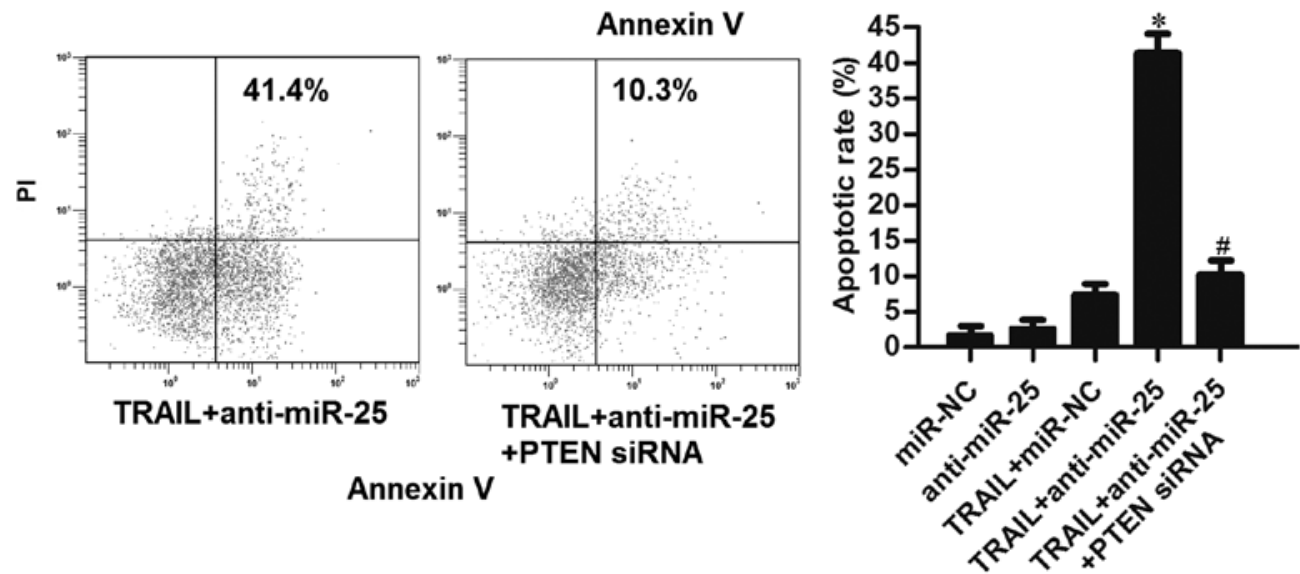

B

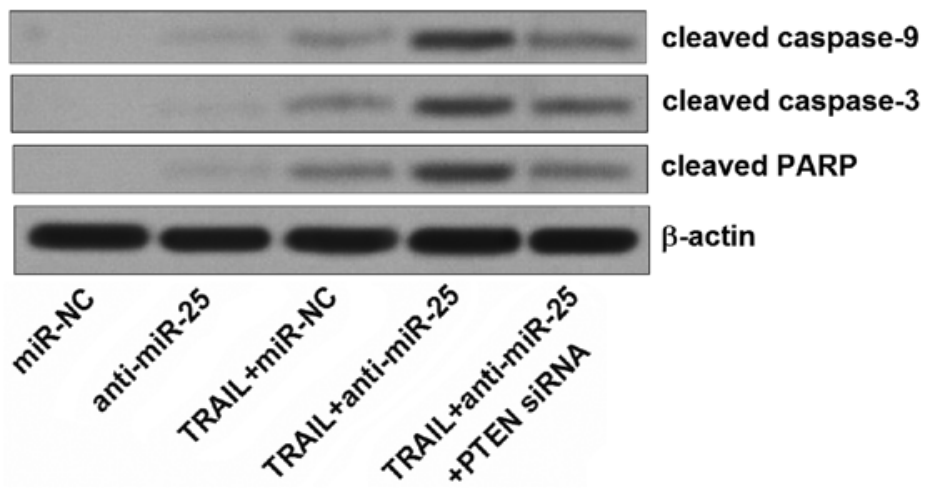

Figure 6. Knockdown of miR-25 in HepG2-CSCs enhanced the tumor necrosis factor (TNF)-related apoptosis inducing ligand (TRAIL)-induced apoptosis through the phosphatase and tensin homologue (PTEN) pathway. (A) The anti-miR-25 enhanced the TRAIL (10 ng/ml)-induced apoptosis in HepG2-CSCs, which could be inhibited by the transfection of PTEN siRNA. "P $<0.05$ vs. TRAIL + miR-NC group; ${ }^{\text {P }}<0.05$ vs. TRAIL + anti-miR- 25 group. (B) The cleavage of caspase-9, caspase-3 and its substrate of PARP was evaluated by western blot analysis after the HepG2-CSCs were treated with anti-miR-25, TRAIL and PTEN siRNA.

Knockdown of miR-25 enhances the TRAIL-induced apoptosis via PTEN/PI3K/Akt/Bad signaling pathway. Since the PTEN is a natural inhibitor of PI3K and negatively regulates the Akt (16), we next investigated the relationship between the anti-miR-25 and the PTEN/PI3K/Akt signaling. Transfection of anti-miR-25 inhibited the phosphorylation of PI3K and Akt (Fig. 7A). In contrast, the single TRAIL treatment did not alter the activity of PI3K or Akt. Previous studies have demonstrated that Bad is one of the substrates for Akt (17). As we observed, the anti-miR-25 induced significant inhibition of Bad phosphorylation, which may be the molecular mechanism by which the anti-miR-25 promotes the apoptosis in HepG2-CSCs (18). The dephosphorylation of Bad induced by the anti-miR-25, knockdown of miR-25 significantly increased the Bad-Bcl-2 heterodimer and the Bad-Bcl-xL heterodimer in HepG2-CSCs (Fig. 7B), subsequently inactivating the anti-apoptotic proteins of Bcl-2 and $\mathrm{Bcl}-\mathrm{xL}$. Studies have demonstrated that the inactivation of $\mathrm{Bcl}-2$ and $\mathrm{Bcl}-\mathrm{xL}$ promotes the release of mitochondria-derived apoptogenic compounds (19). We therefore detected the cytochrome $c$, Smac/DIABLO, and AIF in the cytoplasm of HepG2-CSCs. The levels of all of these apoptogenic compounds were significantly increased in the cytoplasm after the HepG2-CSCs were co-treated with TRAIL and anti-miR-25 (Fig. 7C). In addition, ROS which is an important apoptosis inducer (20) was also generated after the combination treatment with TRAIL and anti-miR-25 (Fig. 7D). Taken together, our data strongly suggests that the anti-miR-25 promotes the TRAIL-induced apoptosis which is regulated by the PTEN/PI3K/Akt/Bad signaling pathway. 

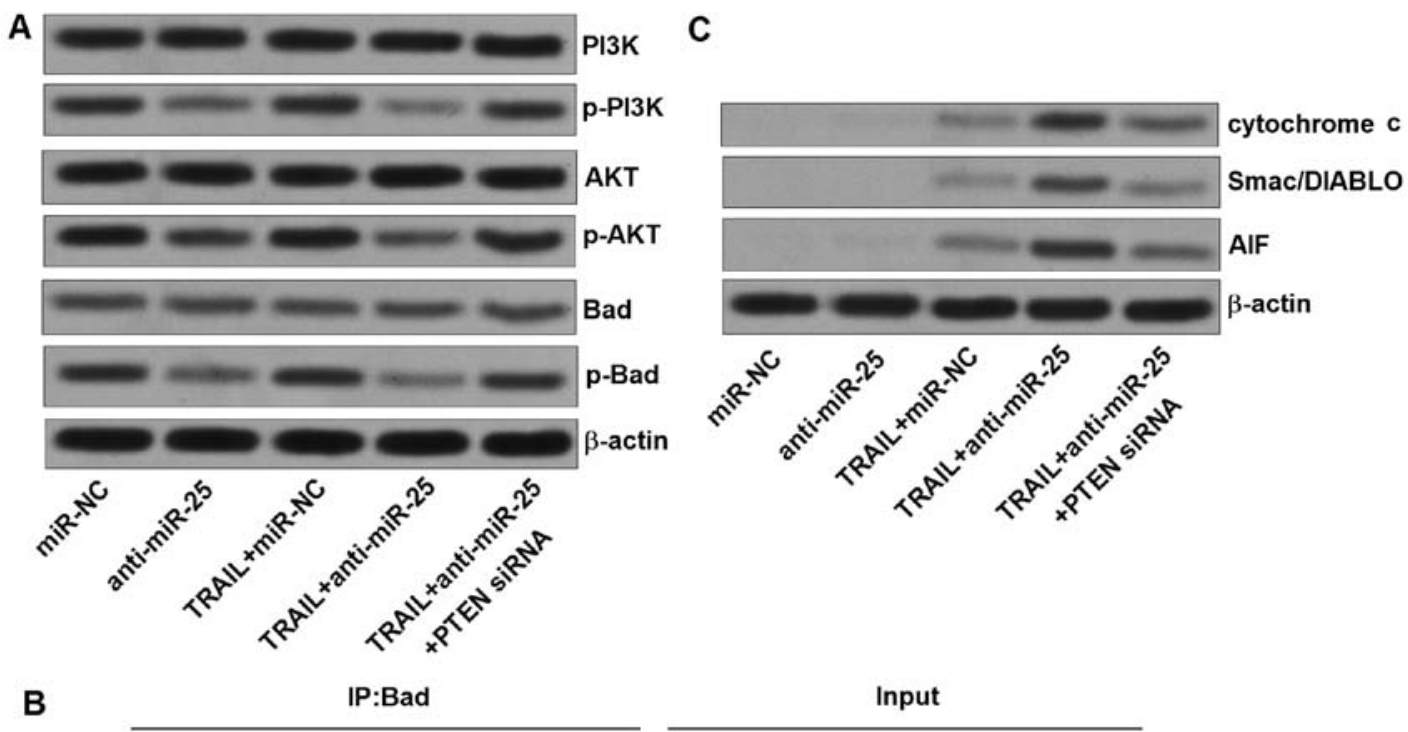

B

IP:Bad
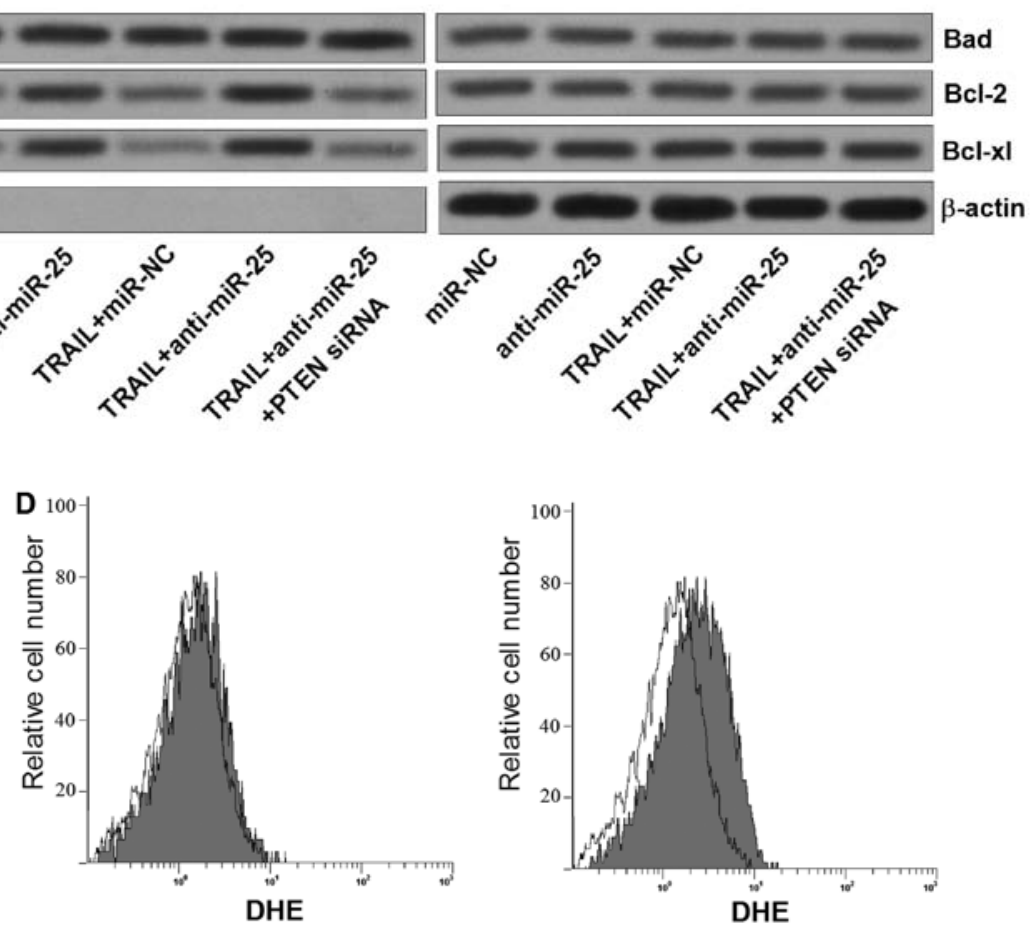

$\square$ miR-NC

anti-miR-25



$\square$ miR-NC

TRAIL+miR-NC

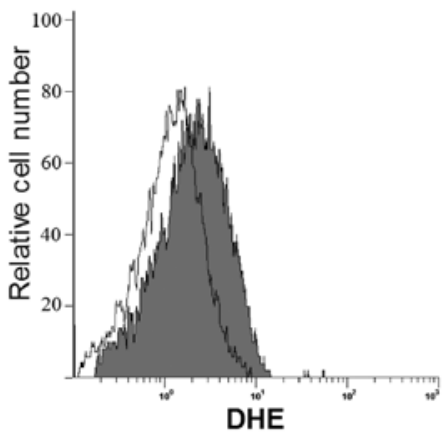

$\square$ miR-N

TRAIL+anti-miR-25



Figure 7. Knockdown of miR-25 enhances the tumor necrosis factor (TNF)-related apoptosis inducing ligand (TRAIL)-induced apoptosis via phosphatase and tensin homologue (PTEN)/PI3K/Akt/Bad signaling pathway. (A) Western blot analysis was performed to evaluate the phosphorylation of PI3K, Akt and Bad in HepG2-CSCs treated with anti-miR-25, TRAIL (10 ng/ml), and PTEN siRNA. (B) Co-immunoprecipitation was performed to detect the Bad-Bcl-2 heterodimer and the Bad-Bcl-xL heterodimer in HepG2-CSCs treated with anti-miR-25, TRAIL and PTEN siRNA. (C) After the mitochondria were removed, the proteins of cytochrome $c$, Smac/DIABLO and AIF in the cytoplasm of HepG2-CSCs were detected by western blot analysis. (D) The generation of reactive oxygen species (ROS) in HepG2-CSCs was measured by dihydroethidium (DHE) staining. 


\section{Discussion}

Previous studies have demonstrated that miRNAs are associated with the sensitivity of cancer cells to the antitumor treatment. For instance, overexpression of miR-122 in HCC could reverse the resistance to doxorubicin by inhibiting the glucose metabolism of cancer cells (21). Knockdown of miR-221 could enhance the TRAIL-induced apoptosis by upregulating the expression of BIM gene in breast cancer (22). The tumor suppressor of miR-101 could sensitize the HCC cells to the induction of apoptosis via targeting the gene of Mcl-1 (23). It is clear that the dysregulation of miRNAs should be considered responsible for the low-sensitivity of cancer cells to the chemotherapy.

miR-25 has been reported to act as an onco-miRNA in various cancers, such as ovarian cancer, breast cancer and gastric cancer. High levels of miR-25 in plasma predicted poor prognosis in patients with gastric cancer (24-26). However, the role of miR-25 in the cancer stem cells of HCC remains unclear. In the present study, we demonstrate that the miR-25 is dysregulated in the LCSCs. Moreover, we proved that the knockdown of miR-25 could significantly increase the sensitivity of HepG2-CSCs to the treatment of TRAIL in vitro and in vivo. Interestingly, knockdown of miR-25 could inhibit the effect of TRAIL on enriching the HepG2-CSCs population, because the absence of miR-25 promoted the killing of HepG2-CSCs induced by TRAIL. These findings may provide a potential use of miR-25 antagonist as a therapeutic strategy for reducing the drug-resistance of LCSCs.

PTEN is a well-known tumor suppressor in multiple cancers, which inhibits the tumorigenicity by negatively regulating the highly oncogenic PI3K/Akt-signaling pathway (27). Previous studies have demonstrated that the gene of PTEN is dysregulated in various cancers, and the change of PTEN is one of the critical factors for tumorigenesis $(28,29)$. Recently, it was reported that PTEN is regulated by miRNAs in cancers, and the miRNAs/PTEN axis has been confirmed to be associated with the tumor growth, migration, invasion and chemosensitivity (30-32). In this study we also indicated that the gene of PTEN is downregulated in the CSCs of HepG2. Moreover, the miR-25/PTEN axis determined the sensitivity of HepG2-CSCs to TRAIL.

BCL2 associated agonist of cell death (Bad) is a proapoptosis protein belonging to the $\mathrm{Bcl}-2$ family, which is also a substrate of Akt. The non-phosphorylated Bad promotes apoptosis by conjugating to the anti-apoptotic proteins of Bcl-2 and $\mathrm{Bcl}-\mathrm{xL}$, subsequently inactivating them. However, when Bad is phosphorylated by Akt, it will separate from the heterodimer of Bcl-2/Bcl-xL, and bind to 14-3-3 scaffold proteins in the cytoplasm with an inactive form. Thus, the free Bcl-2 and Bcl-xL could inhibit the apoptosis pathway $(18,33)$. Our present study demonstrates that the knockdown of miR-25 increases the expression of PTEN, thus inhibiting the PI3K/Akt signaling pathway followed by keeping the Bad in a non-phosphorylated form. Benefiting from this, TRAIL is able to induce the mitochondrial apoptosis (34) in HepG2-CSCs, leading to the release of mitochondria-derived apoptogenic compounds such as cytochrome $c$, Smac/DIABLO, AIF and the ROS. Finally, the combination with anti-miR-25 and TRAIL induces the cleavage of caspases and the occurrence of apoptosis.
In conclusion, we have provided several pieces of evidence to prove that the miR-25 is associated with the sensitivity of liver cancer stem cells to TRAIL-induced apoptosis. Mechanistically, we demonstrate that the knockdown of miR-25 promotes TRAIL-induced apoptosis by inhibiting the PI3K/Akt/Bad signaling pathway through the miR-25/PTEN axis. The combination with anti-miR-25 and TRAIL may represent a novel strategy for the treatment of LCSCs.

\section{Acknowledgements}

This study is supported by the Medical and Health Technology Plan of Zhejiang Province (grant no. 2013KYB117).

\section{References}

1. Siegel R, Naishadham D and Jemal A: Cancer statistics, 2013. CA Cancer J Clin 63: 11-30, 2013.

2. Villanueva A, Newell P, Chiang DY, Friedman SL and Llovet JM: Genomics and signaling pathways in hepatocellular carcinoma. Semin Liver Dis 27: 55-76, 2007.

3. Reya T, Morrison SJ, Clarke MF and Weissman IL: Stem cells, cancer, and cancer stem cells. Nature 414: 105-111, 2001.

4. Clarke MF and Fuller M: Stem cells and cancer: Two faces of eve. Cell 124: 1111-1115, 2006.

5. Suetsugu A, Nagaki M, Aoki H, Motohashi T, Kunisada T and Moriwaki H: Characterization of $\mathrm{CD} 133^{+}$hepatocellular carcinoma cells as cancer stem/progenitor cells. Biochem Biophys Res Commun 351: 820-824, 2006.

6. Ma S, Lee TK, Zheng BJ, Chan KW and Guan XY: CD133+ $\mathrm{HCC}$ cancer stem cells confer chemoresistance by preferential expression of the Akt/PKB survival pathway. Oncogene 27: 1749-1758, 2008.

7. Joshi P, Jeon YJ, Laganà A, Middleton J, Secchiero P, Garofalo M and Croce CM: MicroRNA-148a reduces tumorigenesis and increases TRAIL-induced apoptosis in NSCLC. Proc Natl Acad Sci USA 112: 8650-8655, 2015

8. Walczak H, Miller RE, Ariail K, Gliniak B, Griffith TS, Kubin M, Chin W, Jones J, Woodward A, Le T, et al: Tumoricidal activity of tumor necrosis factor-related apoptosis-inducing ligand in vivo. Nat Med 5: 157-163, 1999.

9. Piggott L, Omidvar N, Martí Pérez S, French R, Eberl M and Clarkson RW: Suppression of apoptosis inhibitor c-FLIP selectively eliminates breast cancer stem cell activity in response to the anti-cancer agent, TRAIL. Breast Cancer Res 13: R88, 2011.

10. Bartel DP: MicroRNAs: Target recognition and regulatory functions. Cell 136: 215-233, 2009.

11. Gargalionis AN and Basdra EK: Insights in microRNAs biology. Curr Top Med Chem 13: 1493-1502, 2013.

12. Calin GA and Croce CM: MicroRNA signatures in human cancers. Nat Rev Cancer 6: 857-866, 2006.

13. Ou Y, Zhai D, Wu N and Li X: Downregulation of miR-363 increases drug resistance in cisplatin-treated HepG2 by dysregulating Mcl-1. Gene 572: 116-122, 2015.

14. Livak KJ and Schmittgen TD: Analysis of relative gene expression data using real-time quantitative PCR and the 2(-Delta Delta C(T)) method. Methods 25: 402-408, 2001.

15. Ma H, Yao Q, Zhang AM, Lin S, Wang XX, Wu L, Sun JG and Chen ZT: The effects of artesunate on the expression of EGFR and ABCG2 in A549 human lung cancer cells and a xenograft model. Molecules 16: 10556-10569, 2011.

16. Stambolic V, Suzuki A, de la Pompa JL, Brothers GM, Mirtsos C, Sasaki T, Ruland J, Penninger JM, Siderovski DP and Mak TW: Negative regulation of $\mathrm{PKB} / \mathrm{Akt}$-dependent cell survival by the tumor suppressor PTEN. Cell 95: 29-39, 1998.

17. Brazil DP and Hemmings BA: Ten years of protein kinase B signalling: A hard Akt to follow. Trends Biochem Sci 26: 657-664, 2001.

18. Datta SR, Dudek H, Tao X, Masters S, Fu H, Gotoh Y and Greenberg ME: Akt phosphorylation of BAD couples survival signals to the cell-intrinsic death machinery. Cell 91: 231-241, 1997.

19. Ye L, Yuan G, Xu F, Sun Y, Chen Z, Chen M, Li T, Sun P, Li S and Sun J: The small-molecule compound BM-1197 inhibits the antiapoptotic regulators $\mathrm{Bcl}-2 / \mathrm{Bcl}-\mathrm{xL}$ and triggers apoptotic cell death in human colorectal cancer cells. Tumour Biol 36: 3447-3455, 2015. 
20. Tian X, Zhao L, Song X, Yan Y, Liu N, Li T, Yan B and Liu B HSP27 inhibits homocysteine-induced endothelial apoptosis by modulation of ROS production and mitochondrial caspasedependent apoptotic pathway. BioMed Res Int 2016: 4847874, 2016.

21. Pan C, Wang X, Shi K, Zheng Y, Li J, Chen Y, Jin L and Pan Z: MiR-122 reverses the doxorubicin-resistance in hepatocellular carcinoma cells through regulating the tumor metabolism. PLoS One 11: e0152090, 2016.

22. Ye Z, Hao R, Cai Y, Wang $X$ and Huang G: Knockdown of miR-221 promotes the cisplatin-inducing apoptosis by targeting the BIM-Bax/Bak axis in breast cancer. Tumour Biol 37: 4509-4515, 2016.

23. He H, Tian W, Chen H and Deng Y: MicroRNA-101 sensitizes hepatocellular carcinoma cells to doxorubicin-induced apoptosis via targeting Mcl-1. Mol Med Rep 13: 1923-1929, 2016.

24. Feng S, Pan W, Jin Y, Zheng J: MiR-25 promotes ovarian cancer proliferation and motility by targeting LATS2. Tumour Biol 35: 12339-12344, 2014.

25. Wang Z, Wang N, Liu P, Chen Q, Situ H, Xie T, Zhang J, Peng C, Lin Y and Chen J: MicroRNA-25 regulates chemoresistanceassociated autophagy in breast cancer cells, a process modulated by the natural autophagy inducer isoliquiritigenin. Oncotarget 5: 7013-7026, 2014.

26. Li BS, Zhao YL, Guo G, Li W, Zhu ED, Luo X, Mao XH, Zou QM, Yu PW, Zuo QF, et al: Plasma microRNAs, miR-223, miR-21 and miR-218, as novel potential biomarkers for gastric cancer detection. PLoS One 7: e41629, 2012.

27. Li J, Yen C, Liaw D, Podsypanina K, Bose S, Wang SI, Puc J, Miliaresis C, Rodgers L, McCombie R, et al: PTEN, a putative protein tyrosine phosphatase gene mutated in human brain, breast, and prostate cancer. Science 275: 1943-1947, 1997.
28. Tay Y, Kats L, Salmena L, Weiss D, Tan SM, Ala U, Karreth F, Poliseno L, Provero P, Di Cunto F, et al: Coding-independent regulation of the tumor suppressor PTEN by competing endogenous mRNAs. Cell 147: 344-357, 2011.

29. Xu W, Yang Z, Zhou SF and Lu N: Posttranslational regulation of phosphatase and tensin homolog (PTEN) and its functional impact on cancer behaviors. Drug Des Devel Ther 8: 1745-1751, 2014.

30. Wu W, Yang J, Feng X, Wang H, Ye S, Yang P, Tan W, Wei G and Zhou Y: MicroRNA-32 (miR-32) regulates phosphatase and tensin homologue (PTEN) expression and promotes growth, migration, and invasion in colorectal carcinoma cells. Mol Cancer 12: 30, 2013.

31. Wang ZX, Lu BB, Wang H, Cheng ZX and Yin YM: MicroRNA-21 modulates chemosensitivity of breast cancer cells to doxorubicin by targeting PTEN. Arch Med Res 42: 281-290, 2011.

32. Ma F, Zhang J, Zhong L, Wang L, Liu Y, Wang Y, Peng L and Guo B: Upregulated microRNA-301a in breast cancer promotes tumor metastasis by targeting PTEN and activating Wnt/ $\beta$-catenin signaling. Gene 535: 191-197, 2014.

33. Datta SR, Brunet A and Greenberg ME: Cellular survival: A play in three Akts. Genes Dev 13: 2905-2927, 1999.

34. Lim SC, Parajuli KR and Han SI: The alkyllysophospholipid edelfosine enhances TRAIL-mediated apoptosis in gastric cancer cells through death receptor 5 and the mitochondrial pathway. Tumour Biol 37: 6205-6216, 2016. 